\title{
Automatic Glitch Elimination of Scanning Probe Microscopy Images
}

\author{
Yuhang CHEN" and Wenhao HuAng \\ Department of Precision Machinery and Precision Instrumentation, University of Science and Technology of China, \\ Hefei 230026, P. R. China
}

\begin{abstract}
Glitch artifacts appear in many scanning probe microscopy (SPM) images due to transient instabilities. Such artifacts can distort any quantitative analyses based on the measured images. A robust smoothing method has been adopted to eliminate the glitch artifacts. Results on different kinds of sample images demonstrate that this method is quite efficient. However, the smoothing operation will cause information loss in certain cases, especially on sharp structures. If these features are also crucial in analysis, the compromise between the elimination of glitches and the reservation of signal details should be settled. Post-restoration of image details from analyzing extracted glitch artifacts can meet this demand. Glitch elimination can help to improve the value of SPM in quantitative nanoscale measurements.
\end{abstract}

(Received July 28, 2010; Accepted November 16, 2010; Published February 10, 2011)

\section{Introduction}

Scanning probe microscopy (SPM) has been adopted as a powerful technique in the fields of nanoscience and nanotechnology. Due to the ultrahigh resolution, SPM has been widely used for the visualization of various surface structures at a nanoscale. ${ }^{1}$ However, practical SPM images always contain many types of artifacts, such as those induced by tip dilation, ${ }^{2,3}$ imperfect scanner behavior (hysteresis, creep and nonlinearity), ${ }^{4}$ dynamic properties of the microscope, ${ }^{5} \mathrm{drift},{ }^{6,7}$ and so on. All of these artifacts will distort the measurements, especially in cases where quantitative evaluations are necessary.

In this work, we concentrate on an investigation of the glitch artifact, which appears occasionally in experiments. The glitches are actually line-patterns with extreme values compared with their neighbor data in the image. ${ }^{8}$ They are mainly introduced by random instabilities during the acquisition of images. In the case of atomic force microscopy (AFM), several experimental factors can contribute to the glitch artifact: 1) Surface contamination. When either the tip or the sample is contaminated, the AFM tip may slip on the surface due to high friction forces. This slip can cause a mis-response of the scanner elongation, and thus a glitch artifact is induced. 2) Uneven adhesion distribution. When the dynamic operation mode (for example, tapping mode) of AFM is adopted, the dramatic changes of the tip-sample adhesion force may alter the oscillation signals, such as the frequency and the vibration amplitude. ${ }^{9}$ These transient responses of the dynamic vibrations, which are used for feedback, will cause sudden piezo-scanner elongations. As a result, the scanned image may contain certain glitches that are not relevant with the sample topography. 3) Intrinsic bi-stability of the nonlinear probe dynamics. Due to the coupling of attractive and repulsive interactions between the

$\dagger$ To whom correspondence should be addressed.

E-mail: chenyh@ustc.edu.cn tip and a sample, the probe dynamics show an intrinsic bi-stability. The switch between the attractive force dominant region and the repulsive force dominant region can induce transient instabilities. ${ }^{10}$ Consequently, the glitch artifact appears in the obtained results. From all of these aspects, such kinds of artifacts are difficult to be eliminated experimentally, since they are sensitive to various complex conditions. The experimental parameters used to reduce a glitch artifact are difficult to be well controlled.

In quantitative analysis based on a measured SPM image, a glitch artifact can certainly have a negative influence. It will distort the results, since glitches are extreme values compared with the real surface information. In a reduction of glitches by off-line image processing, generally known and efficient algorithms to handle this kind of artifact is lacking. ${ }^{8}$ The glitches will persist even after applying most conventional image-processing methods, for example, flatten, smoothing or equalization. Some SPM software programs have provided a manual selection of glitches, and consequent elimination is obtained by a simple local average. The major drawback of the manual approach is that it is usually slow and labor intensive. For the automatic elimination of glitch artifacts, Hyon et al. proposed an algorithm by analyzing the probability distribution of the derivative of the scanned image. ${ }^{8}$ However, their approach is based on the one-dimensional positioning of the glitches, and proper choice of the threshold is also needed. In addition, the differential operation may be sensitive to image noise. Due to all these reasons, we focus on the fully automatic and robust elimination of the glitch artifact in practical SPM images.

\section{Experimental}

All of the experiments presented here were carried out on a commercial AFM system (Nanotec, Spain) in an ambient environment. ${ }^{11}$ The cantilevers were silicon nitride AFM cantilevers with a nominal spring constant of $0.73 \mathrm{~N} / \mathrm{m}$ 

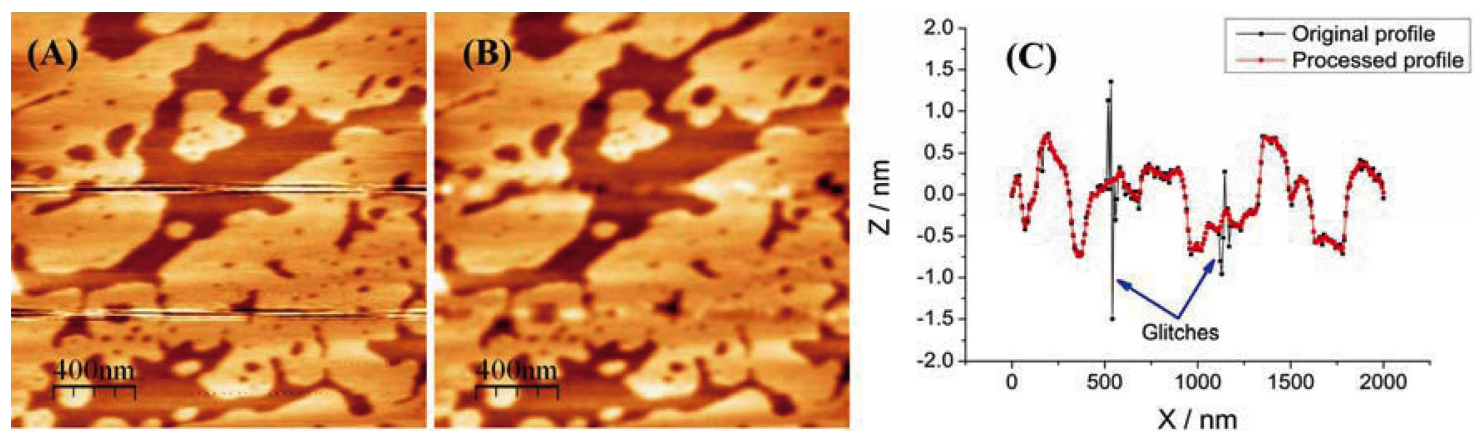

Fig. 1 Glitch elimination on an AFM image of DPPC monolayer. (A) Original AFM image of the DPPC monolayer on mica substrate. The scan area is $2 \times 2 \mu \mathrm{m}$ with $256 \times 256$ pixels. (B) Image after glitch elimination. (C) Corresponding sectional profiles. Here, both profiles are taken at the same $\mathrm{x}$-center.

(Olympus RC800-PSA rectangular cantilevers). Several samples were tested. They were a dipalmitoyl phosphatidylcholine (DPPC) monolayer transferred on a fleshly cleaved mica surface, thin gold film evaporated on a piece of silicon wafer and single-walled carbon nanotubes (SWNTs) deposited on a flat silicon substrate, respectively. Both the contact mode and the tapping mode were used for image acquisition. The obtained images with obvious glitches were selected for further artifact elimination by off-line image processing.

\section{Glitch elimination}

From the sectional profiles or the images (as shown in the following sections), the glitches are usually outlying data. That is, the measured heights or other detected physical signals at the glitch-distorted regions are either much larger or much smaller than their neighbor data points. The surface information is then heavily distorted on the glitches. From an intuitive point of view, the elimination of glitch artifacts is actually a problem of smoothing data points where extreme values, or even missing data points, are presented. According to the operation principle of SPM, the measured image is assumed to be equally sampled. In order to eliminate glitch artifacts, we adopt the newly developed algorithm based on a discrete cosine transform (DCT), which is called as the robust smoothing method. ${ }^{12}$ The advantage of the algorithm is that it is a global smoothing, and it is very suitable for handling extreme values or missing values in obtained data. Several subroutines ensure the stability and reliability of the approach via robust smoothing. Firstly, locally weighted analysis is applied. The outliers in the scanned image are given low weights, and data with high quality are assigned high weights. In such a way, the influence of glitches can be reduced as much as possible. Secondly, in order to avoid either over- or under-processing, generalized cross-validation (GCV) is adopted for automatic evaluation of the smoothing process. Lastly, iterative procedures are used to find the optimized parameters for robust smoothing, that is, to find smoothing parameters that can minimize the GCV score. For further details, Ref. 12 shall be referred. The above method is realized by a home-written program. The processed data are then imported into the freeware WSxM for image shading and representation. ${ }^{11}$

\section{Results and Discussion}

Figure 1(A) shows one typical AFM image of the DPPC sample;
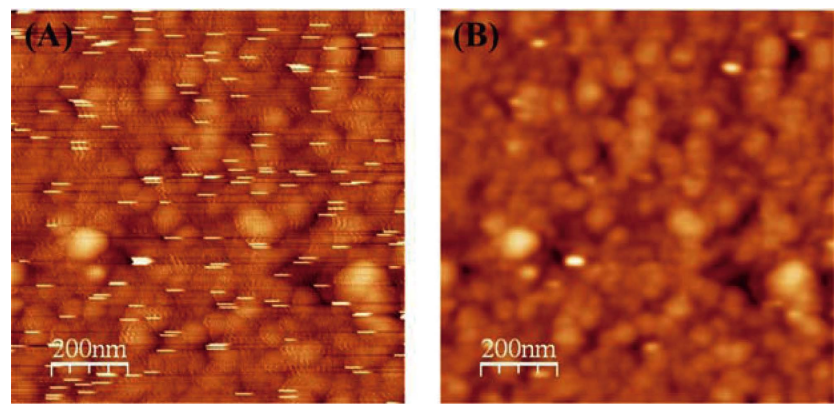

Fig. 2 Application of glitch elimination on an AFM image of gold thin film. (A) Original image with severe glitch distortions. The scan area is $1 \times 1 \mu \mathrm{m}$ with $256 \times 256$ pixels. The image has been applied with a prior offset correction. (B) Image after eliminating the glitches.

the image contains obvious glitches. A monolayer was deposited on the substrate in the liquid expended (LE) and liquid condensed (LC) coexistence region. ${ }^{13}$ The two phases exhibit slight height differences. The sample was prepared by the classic Langmuir-Blodgett technique. From the scanned image, the LE and LC terraces, along with the glitch artifact, are clearly visible. The glitches appear as rough changes of the surface heights. Figure 1(B) depicts the image after artifact elimination. Results demonstrate that the robust smoothing method is very efficient, since the glitch artifact is completely removed. From the central profiles at the same X-center (Fig. 1(C)), the glitches have been thoroughly filtered out while the data outside the glitches maintain almost their original values. The step height between the LE-phase and LC-phase can then be determined via either profile analysis or histogram analysis, without distortions of the glitch artifact. The measured height difference is about $1.1 \mathrm{~nm}$, which is in good agreement with that reported in the literature. ${ }^{14}$

Figure 2(A) shows the AFM image of the gold film on the silicon wafer. Many glitches can be observed, and they tend to appear at the concave areas between the gold particles. Since the experiment is carried out in an ambient environment, it has been demonstrated that higher adhesion force will be present in the concave regions. ${ }^{15}$ As a result, dramatic changes of the oscillation amplitude may occur due to the higher energy dissipation when the vibrating AFM tip passes over these positions. The sharp changes in the dynamic properties consequently cause instabilities of the AFM tip oscillation, and 
so the glitches are introduced in the image. Figure 2(B) shows the image after artifact-elimination processing. From the result, the automatic robust smoothing method is shown to be quite efficient, even on this random rough surface.

The applications on the DPPC image and gold film image demonstrate that robust smoothing removes glitches efficiently on either flat or rough samples. However, the question rises as to whether the processing may cause certain information loss or not. To investigate this issue, we performed robust smoothing on an AFM image of a SWNT sample (Fig. 3(A)). Actually, the
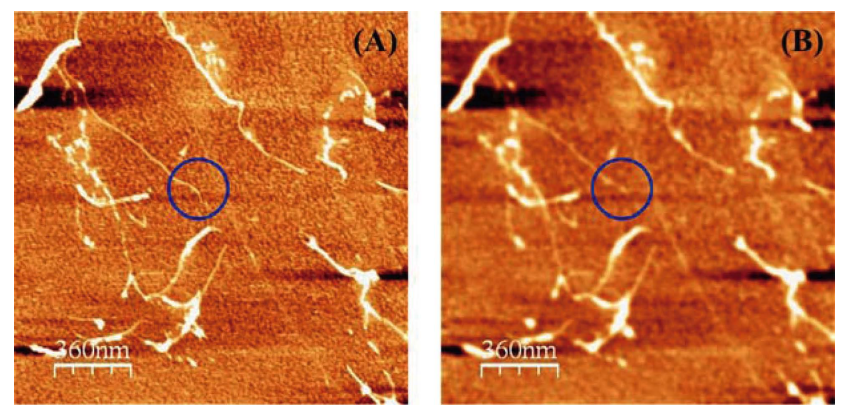

Fig. 3 Adoption of the glitch elimination method on an AFM image of SWNTs on a silicon substrate. (A) Original image. The scan area is $1.8 \times 1.8 \mu \mathrm{m}$ with $512 \times 512$ pixels. Actually, the image has sufficiently high quality without any observable glitches. (B) Image after glitch elimination. Information loss is observed, as indicated by the two circles. image quality was quite good without any visible glitch artifact. Nevertheless, we used the tiny SWNTs on the atomically flat substrate to find out if robust smoothing would cause unacceptable information loss. Figure 3(B) shows the resulting image, and certain distortions on the small structures are found. Compared with the original image, the processed one obviously loses any detailed information at the end section of the SWNT, as indicated by two circles.

If we are only concerned with the main measured surface properties, then robust smoothing has been demonstrated to be sufficiently efficient. If the surface details are also very important, the compromise between artifact elimination and a preservation of signal details should be settled. In fact,
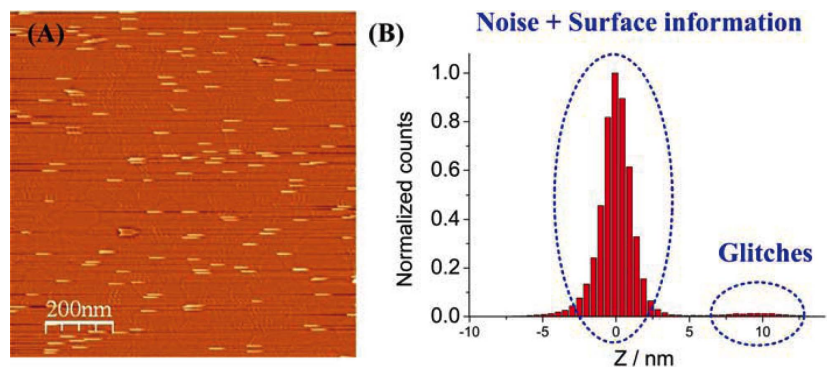

Fig. 4 Distribution properties of glitches analyzed from the extracted artifact image of gold film. (A) Extracted image of the glitch artifact, with shallow surface information. (B) Height histogram of the artifact image.
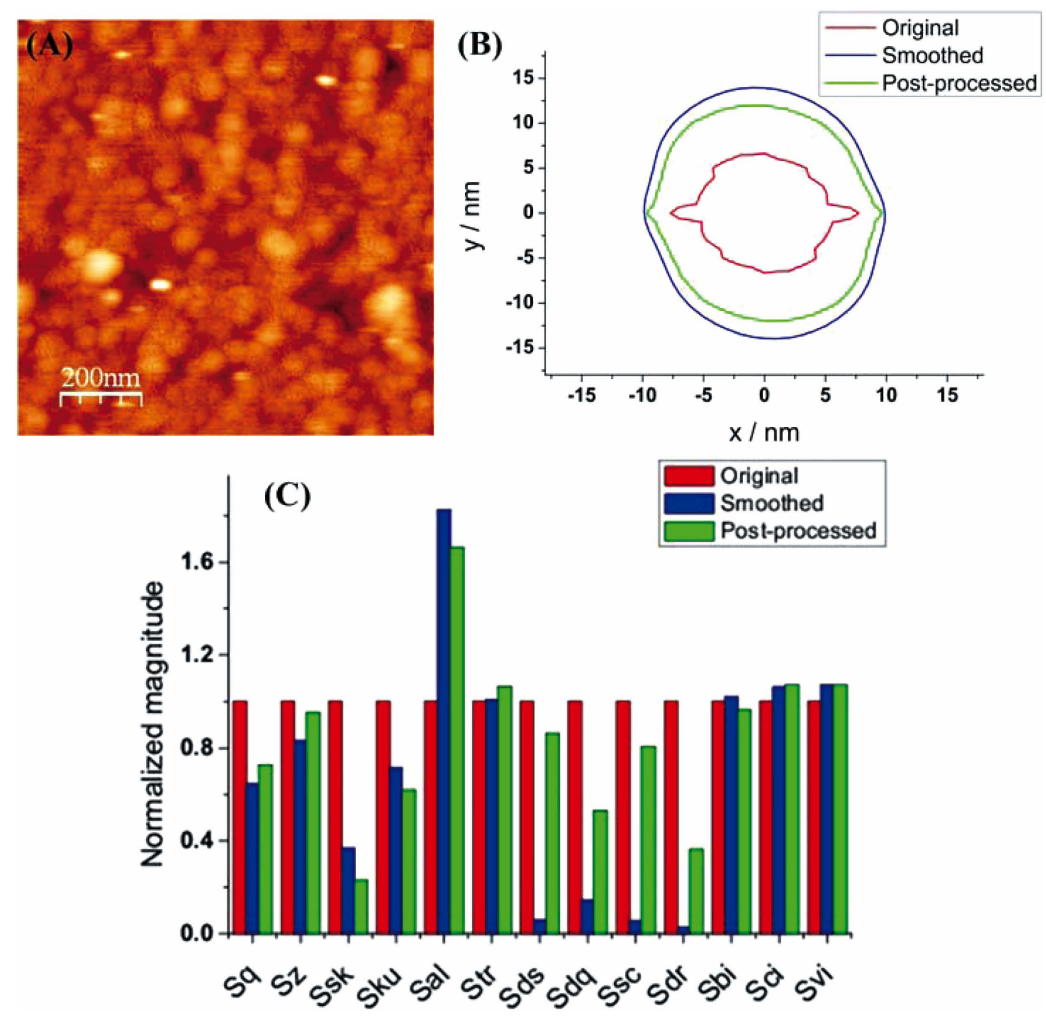

Fig. 5 Comparisons of the original, glitch eliminated and post-processed images of a gold film sample. (A) Image after post-restoration of surface information. (B) Comparison of the central correlation contours of the surface. (C) Comparison of several groups of surface parameters. Here, all surface parameters have been normalized. 
some sacrificed surface information can be persevered by post-restoration. In order to do so, we revisit the case of gold film (Fig. 2). From the extracted image of the glitch artifact (Fig. 4(A)), we can find certain shallow surface information. Then, we do a simple histogram analysis. It can be reasonably assumed that the noise and the remaining surface information will mainly distribute near the central region because the main surface heights have been retracted by robust smoothing, and the AFM image has been applied with a prior offset correction. The distributions located at the two sides in the height histogram are contributed by the glitches in majority. The shallow surface information can then be restored by simple threshold processing with the glitches safely separated. In our experience, the glitches are always distinguishable in the extracted image, since their magnitudes are larger than the noise level. However, it should be mentioned that some amounts of image noise are also recovered by such a process.

Using the post-process, the obtained image is shown in Fig. 5(A). We can see that the remaining surface information, together with a certain amount of noise, is restored. For further quantitative analysis, we analyzed the correlation contour and performed evaluations of the surface parameters from the original, smoothed and post-processed images, respectively. From the correlation contour at 20\% decay (Fig. 5(B)), the original data show inhomogeneous properties, which is not true due to distortions of the glitches. After the smoothing process, the image presents homogenous properties. The correlation lengths in both the $\mathrm{x}$ - and $\mathrm{y}$-directions are almost the same. The correlation length after post-processing is in good agreement with that after pure robust smoothing. They both deviate from the value determined by using the original image.

In quantitative characterizations of the geometrical properties of three-dimensional surfaces, many parameters have already been developed. The most widely used parameters can be divided into four groups: 1) amplitude parameters (root-meansquare (rms) height of surface topography $S_{\mathrm{q}}$, ten-point height $S_{\mathrm{z}}$, skewness of height distribution $S_{\mathrm{sk}}$ and kurtosis of height distribution $S_{\mathrm{ku}}$ ); 2) spatial parameters (density of summits $S_{\mathrm{ds}}$, texture aspect ratio $S_{\mathrm{tr}}$, the fast decay autocorrelation length $S_{\mathrm{al}}$, and texture direction $S_{\mathrm{td}}$ ); 3 ) hybrid parameters (rms slope $S_{\mathrm{dq}}$, mean summit curvature $S_{\mathrm{sc}}$, and developed interfacial area ratio $S_{\mathrm{dr}}$ ); and 4) functional parameters (surface bearing index $S_{\mathrm{bi}}$, core fluid retention index $S_{\mathrm{ci}}$ and valley fluid retention index $S_{\mathrm{vi}}$ ). The major functional surface properties can be described by all of these parameters. Detailed definitions and applications of these parameters can be found in references. ${ }^{16,17}$ Figure 5(C) shows comparison results of the calculated surface parameters. All of the parameters illustrated in the figure have been normalized, that is, they have been divided by values obtained from the original image. The comparison results imply that the amplitude, spatial and hybrid parameters are all greatly distorted by the glitch artifact. Especially, distortions on the evaluations of hybrid parameters are the most dramatic, since they are surface slope or curvature relevant. Though our presented results concentrate on the topographical signal, the main conclusions should still hold for the influences of glitch artifacts on other types of physical signals.

\section{Conclusions}

A robust smoothing method has been adopted to eliminate glitch artifacts in SPM images. Results on several samples with different geometrical properties demonstrate that such an approach is efficient. However, the smoothing process will cause some information loss of sharp surface structures in certain cases. If the sharp features are also important, a compromise between artifact elimination and a reservation of signal details should be settled. This requirement can be satisfied by post-restoration of surface information. Glitch artifacts can introduce great distortions on various surface evaluation parameters, especially the surface slope or curvature relevant parameters. Automatic and robust elimination of glitch artifacts may help to improve the nanoscale quantitative analysis by SPM.

\section{Acknowledgements}

This work was supported by the National Natural Science Foundation of China (No. 50805136).

\section{References}

1. T. Ando, T. Uchihashi, and T. Fukuma, Prog. Surf. Sci., 2008, 83, 337.

2. Y. Chen and W. Huang, Meas. Sci. Technol., 2004, 15, 2005.

3. D. Fujita, H. Itoh, S. Ichimura, and T. Kurosawa, Nanotechnology, 2007, 18, 084002.

4. H. Jin and H. A. Bruck, Nanotechnology, 2005, 16, 1849.

5. Y. Chen and W. Huang, Meas. Sci. Technol., 2010, 21, 045501 .

6. Y. Chen and W. Huang, Rev. Sci. Instrum., 2007, 78, 073701 .

7. C. A. Clifford and M. P. Seah, Meas. Sci. Technol., 2009, 20, 095103.

8. C. Hyon, S. Oh, H. Kim, S. Sull, D. Ahn, Y. Park, and E. Kim, Rev. Sci. Instrum., 2002, 73, 3245.

9. E. Sahagun, P. Garcia-Mochales, G. M. Sacha, and J. J. Saenz, Phys. Rev. Lett., 2007, 98, 176106.

10. S. Santos, V. Barcons, J. Font, and N. H. Thomson, Nanotechnology, 2010, 21, 225710.

11. I. Horcas, R. Fernandez, J. M. Gomez-Rodriguez, J. Colchero, J. Gomez-Herrero, and A. M. Baro, Rev. Sci. Instrum., 2007, 78, 013705.

12. D. Garcia, Comput. Statist. Data Anal., 2010, 54, 1167.

13. C. W. Hollars and R. C. Dunn, Biophys. J., 1998, 75, 342.

14. D. Ebeling, H. Holscher, H. Fuchs, B. Anczykowski, and U. D. Schwarz, Nanotechnology, 2006, 17, S221.

15. P. J. de Pablo, J. Colchero, J. Gomez-Herrero, and A. M. Baro, Appl. Phys. Lett., 1998, 73, 3300.

16. W. P. Dong, P. J. Sullivan, and K. J. Stout, Wear, 1994, 178, 29.

17. W. P. Dong, P. J. Sullivan, and K. J. Stout, Wear, 1994, 178, 45. 